\title{
Impaired type I interferon activity and inflammatory responses in severe COVID-19 patients
}

\begin{abstract}
Jérôme Hadjadji,2*, Nader Yatim ${ }^{2,3 *}$, Laura Barnabei $^{1}$, Aurélien Corneau ${ }^{4}$, Jeremy Boussier $^{3}$, Nikaïa Smith $^{3}$, Hélène Pérés $^{5,6}$, Bruno Charbit $^{7}$, Vincent Bondet ${ }^{3}$, Camille Chenevier-Gobeaux ${ }^{8}$, Paul Breillat ${ }^{2}$, Nicolas Carlier ${ }^{9}$, Rémy Gauzit $^{10}$, Caroline Morbieu ${ }^{2}$, Frédéric Pène ${ }^{11}$, Nathalie Marin $^{11}$, Nicolas Roche ${ }^{9}$, Tali-Anne Szwebel ${ }^{2}$, Sarah H Merkling ${ }^{12}$, Jean-Marc Treluyer ${ }^{13}$, David Veyer ${ }^{5}$, Luc Mouthon², Catherine Blanc ${ }^{4}$, Pierre-Louis Tharaux ${ }^{6}$, Flore Rozenberg' ${ }^{14}$, Alain Fischer,15,16, Darragh Duffy ${ }^{3,7+}$, Frédéric Rieux-Laucat ${ }^{1}+$, Solen Kernéis ${ }^{10,17}+$, Benjamin Terrier $^{2,6+\ddagger}$

1Imagine institute, laboratory of Immunogenetics of Pediatric Autoimmune Diseases, INSERM UMR 1163, Université de Paris, F-75015, Paris. Department of Internal Medicine, National Referral Center for Rare Systemic Autoimmune Diseases, Assistance Publique Hôpitaux de Paris-Centre (APHP-CUP), Université de Paris, F-75014, Paris. ${ }^{3}$ Laboratory of Dendritic Cell Immunobiology, Inserm U1223, Department of Immunology, Institut Pasteur, F-75015, Paris. ${ }^{4}$ Sorbonne Université, Faculté de médecine, UMS037, PASS, Plateforme de cytométrie de la Pitié-Salpêtrière CyPS, F-75013, Paris. ${ }^{5}$ Department of Virology, APHP-CUP, Université de Paris, F-75015, Paris. ${ }^{6}$ PARCC, INSERM U970, Paris. ${ }^{7}$ Cy tometry and Biomarkers UTechS, CRT, Institut Pasteur, F-75015, Paris. ${ }^{8}$ Department of Automated Diagnostic Biology, APHP-CUP, F-75014, Paris. 9Department of Pulmonology, APHP-CUP, Institut Cochin, UMR 1016, Université de Paris, F-75014, Paris. ${ }^{10}$ Equipe Mobile d'Infectiologie, APHP-CUP, Université de Paris, F75014, Paris. ${ }^{11}$ Medical intensive care unit, APHP-CUP, Institut Cochin, INSERM U1016, CNRS UMR 8104, Université de Paris, F-75014, Paris. ${ }^{12}$ Insect-Virus Interactions Unit, Institut Pasteur, UMR2000, CNRS, Paris. ${ }^{13}$ Centre Régional de Pharmacovigilance, APHP-CUP, Université de Paris, F-75014, Paris. ${ }^{14 D e p a r t m e n t ~ o f ~ V i r o l o g y, ~ A P H P-C U P, ~}$ Université de Paris, F-75014, Paris. ${ }^{15}$ Unité d'immunologie hématologie et rhumatologie pédiatriques, APHP-CUP, Université de Paris, F-75015, Paris. ${ }^{16}$ Collège de France, Paris. ${ }^{17}$ Epidémiologie et modélisation de la résistance aux antimicrobiens, Institut Pasteur, F-75015, Paris, France.
\end{abstract}

*These authors contributed equally to this work.

†These authors contributed equally to this work.

†Corresponding author. E-mail: benjamin.terrier@aphp.fr

Coronavirus disease 2019 (COVID-19) is characterized by distinct patterns of disease progression suggesting diverse host immune responses. We performed an integrated immune analysis on a cohort of 50 COVID-19 patients with various disease severity. A unique phenotype was observed in severe and critical patients, consisting of a highly impaired interferon (IFN) type I response (characterized by no IFN- $\beta$ and low IFN- $\alpha$ production and activity), associated with a persistent blood viral load and an exacerbated inflammatory response. Inflammation was partially driven by the transcriptional factor NF- $\mathrm{B}$ and characterized by increased tumor necrosis factor (TNF)- $\alpha$ and interleukin (IL)- 6 production and signaling. These data suggest that type-I IFN deficiency in the blood could be a hallmark of severe COVID-19 and provide a rationale for combined therapeutic approaches.

Early clinical descriptions of the first SARS-CoV-2 coronavirus disease (COVID-19) cases at the end of 2019 rapidly highlighted distinct patterns of disease progression (1). Although most patients experience mild-to-moderate disease, 5$10 \%$ progress to severe or critical disease, including pneumonia and acute respiratory failure $(2,3)$. Based on data from patients with laboratory-confirmed COVID-19 from mainland China, admission to intensive care unit (ICU), invasive mechanical ventilation or death occurred in $6.1 \%$ of cases (1), and the death rate from recent current French data was $0.70 \%$ (3). This proportion of critical cases is higher than that estimated for seasonal Influenza (4). Additionally, relatively high rates of respiratory failure were reported in young adults (aged 50 years and lower) with previously mild comorbidities (e.g., hypertension, diabetes mellitus, overweight) (5). Severe cases can occur early in the disease course but clinical observations typically describe a two-step disease progression, starting with a mild-to-moderate presentation, followed by a secondary respiratory worsening 9-12 days after the first onset of symptoms $(2,6,7)$. Respiratory deterioration is concomitant with extension of ground-glass lung opacities on chest computed tomography (CT) scans, lymphocytopenia, high prothrombin time and D-dimer levels (2). This biphasic evolution marked by a dramatic increase of acute phase reactants in the blood suggests a dysregulated inflammatory host response resulting in an imbalance between pro- and antiinflammatory mediators. This leads to the subsequent recruitment and accumulation of leukocytes in tissues causing acute respiratory distress syndrome (ARDS) (8). However, little is known about the immunological features and the molecular mechanisms involved in COVID-19 severity. 
To test the hypothesis of a virally-driven hyperinflammation leading to severe disease, we employed an integrative approach based on clinical and biological data, in-depth phenotypical analysis of immune cells, standardized wholeblood transcriptomic analysis and cytokine measurements on a group of fifty COVID-19 patients with variable severity from mild to critical.

COVID-19 patients $(n=50)$ and healthy controls $(n=18)$ were included. Patients' characteristics are detailed in the supplementary materials and depicted in table S1 and fig. S1. Patients were analyzed after a median duration of 10 days (interquartile range, 9 -11 days) after disease onset. On admission, the degree of severity of COVID-19 was categorized as mild-to-moderate ( $n=15$ patients), severe ( $n=17$ patients) and critical ( $n=18$ patients).

As reported in previous studies $(1,2,8)$, lymphocytopenia correlates with disease severity (Fig. 1A). To further characterize this, we used mass cytometry and performed Visualization of t-Distributed Stochastic Neighbor Embedding (viSNE) (9) to compare cell population densities according to disease severity (Fig. 1B). viSNE representation and differentiated cell counts showed a decrease in the density of NK cells and $\mathrm{CD}^{+} \mathrm{T}$ cells, including all $\mathrm{T}$ cell subsets, that was more pronounced for $\mathrm{CD}^{+} \mathrm{T}$ cells. This phenotype was more prominent in severe and critical patients, contrasting with an increase in the density of B cells and monocytes (Fig. 1, C to F). No major imbalance in $\mathrm{CD}_{4}^{+}$and $\mathrm{CD}^{+} \mathrm{T}$ cell naïve/memory subsets was observed (fig. S2). Data on T cell polarization and other minor $\mathrm{T}$ cell subsets are shown in fig. S3. Plasmablasts were enriched in all infected patients (Fig. 1F), as supported by the increase in genes associated with B cell activation and plasmablast differentiation, such as $I L 4 R$, TNFSF13B and XBP1 (fig. S4) but without any significant increase of serum immunoglobulin levels (fig. S5).

We then assessed the functional status of specific $\mathrm{T}$ cell subsets and NK cells using markers of activation (CD25, CD38, HLA-DR) and exhaustion (PD-1, Tim-3) (fig. S6A). The $\mathrm{CD}^{+}$and $\mathrm{CD}^{+} \mathrm{T}$ cell populations were characterized by an increase in $\mathrm{CD} 38^{+} \mathrm{HLA}^{-\mathrm{DR}^{+}}$activated $\mathrm{T}$ cells in all infected patients, with an expression of PD-1 moderately increasing with disease severity (Fig. $1 \mathrm{G}$ and fig. S6B). A similar increase in activated NK cells was found in all infected patients, especially critical patients, and NK cells displayed a significant increase in Tim-3 expression (Fig. 1G). Furthermore, expression of exhaustion-related genes, such as BATF, IRF4 and CD274, significantly increased with disease severity (fig. S6C). High annexin-V expression (by flow cytometry) and up-regulation of apoptosis-related genes in the blood from severe and critical patients supported the notion that lymphocytopenia could be partly explained by exacerbated $\mathrm{T}$ cell apoptosis (fig. S7).

First release: 13 July 2020
To investigate the immunological transcriptional signatures that characterize disease severity, we quantified the expression of immune-related genes in peripheral white blood cells (Fig. 2A). We identified differentially expressed genes as a function of severity grades (Fig. 2B). Unsupervised principal component analysis (PCA) separated patients with high disease severity on principal component 1 (PC1), driven by inflammatory and innate immune response encoding genes (GSEA enrichment score with q-value <0.2) (Fig. 2C). PC2, that was enriched in genes encoding proteins involved in both type I and type II interferon (IFN) responses, distinguished mild-to-moderate patients from the other groups. Collectively, these data suggested a severity grade-dependent increase in activation of innate and inflammatory pathways; in contrast, the IFN response was high in mild-to-moderate patients while it was reduced in more severe patients.

Type I IFNs are essential for antiviral immunity (10). Multiplex gene expression analysis showed an up-regulation of genes involved in type I IFN signaling (such as IFNARI, JAKI, TYK2) contrasting with a striking down-regulation of interferon-stimulated genes (ISGs) (such as MX1, IFITM1, IFIT2) in critical SARS-CoV-2 patients (Fig. 3A). Accordingly, a validated ISG score, based on the mean of expression of 6 ISGs defining a type I IFN signature (11), was significantly reduced in critical patients compared with patients that had mild-tomoderate infection (Fig. 3B and fig. S8A). IFN- $\beta$ mRNA was undetectable in all infected patients (fig. S8B) as well as IFN$\beta$ protein (fig. S8C). Consistent with ISG scores, plasma levels of IFN- $\alpha 2$ protein measured by Simoa digital ELISA (12) were significantly lower in critical than in mild-to-moderate patients (Fig. 3C) and correlated with ISG $\left(R^{2}=0.30 ; P<0.0001\right)$ (fig. S8D). This result apparently contrasted with the increased detection of IFNA2 mRNA in most severe patients, albeit at levels just above the limit of detection (fig. S8E). To assess the global type I IFN activity, an in vitro cytopathic assay was used (13). IFN activity in serum was significantly lower in severe or critical patients as compared to mild-tomoderate patients (Fig. 3D). ISG score and plasma levels of IFN- $\alpha 2$ from blood collected prior to respiratory failure requiring mechanical ventilation revealed that the low type I IFN response preceded clinical deterioration to critical status (Fig. 3E). Furthermore, low plasma levels of IFN- $\alpha 2$ was significantly associated with an increased risk of evolution to critical status (OR 12, 95\% CI 1.21-118, $P=0.03$ ). Interestingly, analysis in patients where multiple time points were available showed distinct patterns of IFN- $\alpha$ production with sustained high response in mild-to-moderate patients, high but short response in severe patients, and low or no response in critical patients (Fig. 3F). Of note, the proportion of plasmacytoid dendritic cells, the main source of IFN- $\alpha$ (14), was reduced in infected patients compared to healthy controls, possibly due to migration to sites of infection (15), but 
without any difference between groups (Fig. 3G). We next evaluated the response of whole blood cells to IFN- $\alpha$ stimulation (11) and observed a comparable increase in ISG score upon IFN- $\alpha$ stimulation between groups of any severity and controls (Fig. $3 \mathrm{H}$ ), suggesting that the potential for response to type I IFN was not impacted in COVID-19 patients. As a possible consequence of impaired IFN- $\alpha$ production, we used ultrasensitive droplet based digital PCR (ddPCR) and found an increased plasma viral load in severe and critical patients, a possible surrogate marker of uncontrolled lung infection, while viral load in nasal swabs using classical RT-PCR was comparable between groups (Fig. 3I). Overall, these data suggest that infected patients had no detectable circulating IFN$\beta$ and that an impaired IFN- $\alpha$ production characterized the most severe COVID-19 cases.

Severe COVID-19 was reported to be associated with hypercytokinaemia $(8,16)$. Cytokine and chemokine-related genes were found to be increasingly expressed as a function of disease severity in the study cohort (Fig. 4A and fig. S9A). Interestingly, cytokine whole blood RNA levels did not always correlate with protein plasma levels. IL-6, a key player of the exacerbated inflammatory response in COVID-19 (17), was not detected in peripheral blood at the transcriptional level (fig. S9B), contrasting with high levels of IL-6 protein (Fig. 4B). Expression of IL-6-induced genes, such as IL6R, SOCS3 and STAT3 were significantly increased (fig. S9B) reflecting the activation of the IL- 6 signaling pathway. TNF- $\alpha$, a key driver of inflammation, was only moderately up-regulated at the transcriptional level (fig. S9C), whereas circulating TNF$\alpha$ was significantly increased (Fig. 4C). Accordingly, TNF pathway-related genes were also up-regulated, including TNFSF10 (fig. S9, D and E), supporting an important role for TNF- $\alpha$ in the induction of inflammation. The discrepancy between RNA quantification and protein measurement suggests that cellular sources of TNF- $\alpha$ and IL- 6 may be the injured lungs and/or endothelial cells. Conversely, while $I L 1 B$ transcripts were significantly up-regulated (fig. S9F), the active form of IL-1 $\beta$ protein was low (Fig. 4D), suggesting that pro-IL-1 $\beta$ was poorly cleaved and secreted, but does not exclude a local production in the lung (15). Circulating IL-1 $\alpha$ was also not detected (fig. S9F). These findings contrasted with the detection of high levels of circulating IL-1 receptor antagonist (IL-1RA) and up-regulation of ILIRI transcripts, indicating an active antagonism of IL-1 in critically ill patients (fig. S9F). We also detected ILIO transcripts and IL-10 protein in both severe or critical patients (Fig. 4E and fig. S9G). IFN- $\checkmark$ was increased in mild-to-moderate patients and at a lesser extent in severe patients, but not in critical patients. In contrast, no increase in IL-17A levels was detected in all infected patients' groups (fig. S10).

We next explored the expression of transcription factors that may drive this exacerbated inflammation and found that genes specifically up-regulated in severe or critical patients mainly belonged to the NF- $\mathrm{kB}$ pathway (Fig. $4 \mathrm{~F}$ and fig. S11, A and B). Among several triggering pathways, aberrant NF$\kappa B$ activation can result, from excessive innate immune sensor activation by pathogen-associated molecular patterns (PAMPs) (e.g., viral RNA) and/or damage-associated molecular patterns (DAMPs) (e.g., released by necrotic cells and tissue damage). Interestingly, $\mathrm{LDH}$, a marker of necrosis and cellular injury, correlated with disease severity (fig. S1C), and receptor-interacting protein kinase (RIPK)-3, a key kinase involved in programmed necrosis and inflammatory cell death, was also significantly elevated in severe or critical patients (Fig. 4G) and correlated with LDH $\left(R^{2}=0.47 ; P<0.0001\right)$.

The exacerbated inflammatory response has been associated with a massive influx of innate immune cells, namely neutrophils and monocytes, which may aggravate lung injury and precipitate ARDS (15). We therefore analyzed expression of chemokines and chemokine receptors involved in the trafficking of innate immune cells (Fig. 4A). While the neutrophil chemokine CXCL2 was detected in the serum but with no difference between groups, its receptor CXCR2 was significantly up-regulated in severe and critical patients (Fig. $4 \mathrm{H})$. Consistently, severe disease was accompanied with higher neutrophilia (Fig. 4H). Of note, the inflammatory response pattern remained elevated even after normalization of transcriptional data with neutrophil counts (fig. S12). Monocyte chemotactic factor CCL2 was increased in the blood of infected patients, as well as the transcripts of its receptor CCR2; this was associated with low circulating inflammatory monocytes counts (Fig. 4I), suggesting a role for the CCL2/CCR2 axis in the monocyte chemoattraction into the inflamed lungs. These observations are in accordance with published studies in bronchoalveolar fluids from COVID-19 patients, describing the key role of monocytes (15). Overall, these results support a framework whereby an ongoing inflammatory cascade, in the setting of impaired type I IFN production and high viral load may be fueled by both PAMPs and DAMPs.

In this study, we identified an impaired type I IFN response in severe and critical COVID-19 patients, accompanied by high blood viral load and an excessive NF- $\mathrm{k}$-driven inflammatory response associated with increased TNF- $\alpha$ and IL-6. Innate immune sensors, such as TLRs and RIG-I-like receptors, play a key role in controlling RNA virus by sensing viral replication and by alerting the immune system through the expression of a diverse set of antiviral genes (18). Type I IFNs, which include IFN- $\alpha, \beta$ and $\omega$, are hence rapidly induced and orchestrate a coordinated antiviral program via the JAK-STAT signaling pathway and expression of ISGs (19). We observed that SARS-CoV-2 infection was characterized by an absence of circulating IFN- $\beta$ in COVID-19 patients with all disease-severity grades. In addition, most severe COVID-19 patients displayed impaired IFN- $\alpha$ production that was 
associated with lower viral clearance. Interestingly, this low type I IFN signature was similar to that observed in young children with severe, but not mild, respiratory syncytial virus infection (20), but was remarkably different from the transcriptional response induced by other respiratory viruses such as human parainfluenza virus 3 or influenza A virus, both characterized by a stronger type I IFN response in in vitro experiments (21). Importantly, although our study was not designed for longitudinal analyses, we observed that low IFN- $\alpha$ plasma levels preceded clinical deterioration and transfer to ICU and that distinct patterns of circulating IFN$\alpha$ characterized each disease-grade. Formal longitudinal studies will be necessary to dissect type I IFN dynamics during SARS-CoV-2 infection. It will be important to assess in severe and critical COVID-19 patients whether this reduced type I IFN production is present at the onset of infection, whether the production is delayed, or whether IFN production is exhausted after an initial peak. Recent data confirmed in cellular and animal models that SARS-CoV-2 inhibited type I and III induction (21). These results suggest that SARS-CoV2 has developed efficient mechanisms to shut down host IFN production.

Conversely on the host side, several hypotheses may be proposed to explain variability in type I IFN responses to infection. Comorbidities are risk factors for severe COVID-19 that could negatively impact IFN production, and in contrast exacerbate inflammatory responses $(22,23)$. Genetic susceptibility can be also suspected since monogenic disorders in children (24) or susceptibility variants in adults (25), each involving the type I IFN pathway, have been associated with life-threatening influenza infections. Identification of patients with insufficient IFN production, but preserved cellular response to type I IFN could define a high-risk population who might benefit from IFN- $\alpha$ or $-\beta$ treatment. Benefit and risk as well as the best time window for efficacy of IFN administration require to be nevertheless weighed. Alternatively, IFN- $\lambda$ (Type III IFN) could be tested as recently proposed (26), as the receptor is localized on epithelial cells, which may avoid potential adverse effects caused by type I IFN.

Viral replication within the lungs in conjunction with an increased influx of innate immune cells mediates tissue damage and may fuel an auto-amplification inflammatory loop, including targetable production of IL-6 (27) and TNF- $\alpha$ (28), potentially driven by NF- $\mathrm{B}$. Our study provides a case for the inhibition of the TNF- $\alpha$ axis. Indeed, TNF- $\alpha$ is highly expressed in alveolar macrophages and anti-TNF- $\alpha$ does not block immune responses in animal models of viral infection (28).

Our study has some limitations. First, the study was designed as a cross-sectional analysis, although sequential time points were available for some patients. Second, data provided are mainly derived from the blood and do not allow the assessment of immune responses within the lung. In this respect, data from Bost et al. describe a reduced type I IFN signature in BAL macrophages from severe COVID-19 patients, supporting the validity of our analysis (29).

Based on our study, we propose that type I IFN deficiency is a hallmark of severe COVID-19 and infer that severe COVID-19 patients might be potentially relieved from the IFN deficiency by IFN administration and from exacerbated inflammation by adapted anti-inflammatory therapies targeting IL- 6 or TNF- $\alpha$, a hypothesis worth cautious testing.

\section{REFERENCES AND NOTES}

1. W.-J. Guan, Z.-Y. Ni, Y. Hu, W.-H. Liang, C.-Q. Ou, J.-X. He, L. Liu, H. Shan, C.-L. Lei, D. S. C. Hui, B. Du, L.-J. Li, G. Zeng, K.-Y. Yuen, R.-C. Chen, C.-L. Tang, T. Wang, P.Y. Chen, J. Xiang, S.-Y. Li, J.-L. Wang, Z.-J. Liang, Y.-X. Peng, L. Wei, Y. Liu, Y.-H. Hu, P. Peng, J.-M. Wang, J.-Y. Liu, Z. Chen, G. Li, Z.-J. Zheng, S.-Q. Qiu, J. Luo, C.J. Ye, S.-Y. Zhu, N.-S. Zhong; China Medical Treatment Expert Group for Covid-19, Clinical characteristics of coronavirus disease 2019 in China. N. Engl. J. Med. 382, 1708-1720 (2020). doi:10.1056/NEJMoa2002032 Medline

2. C. Huang, Y. Wang, X. Li, L. Ren, J. Zhao, Y. Hu, L. Zhang, G. Fan, J. Xu, X. Gu, Z. Cheng, T. Yu, J. Xia, Y. Wei, W. Wu, X. Xie, W. Yin, H. Li, M. Liu, Y. Xiao, H. Gao, L. Guo, J. Xie, G. Wang, R. Jiang, Z. Gao, Q. Jin, J. Wang, B. Cao, Clinical features of patients infected with 2019 novel coronavirus in Wuhan, China. Lancet 395, 497506 (2020). doi:10.1016/S0140-6736(20)30183-5 Medline

3. H. Salje, C. Tran Kiem, N. Lefrancq, N. Courtejoie, P. Bosetti, J. Paireau, A. Andronico, N. Hozé, J. Richet, C.-L. Dubost, Y. Le Strat, J. Lessler, D. Levy-Bruhl, A. Fontanet, L. Opatowski, P.-Y. Boelle, S. Cauchemez, Estimating the burden of SARS-CoV-2 in France. Science eabc3517 (2020). doi:10.1126/science.abc3517 Medline

4. E. J. Chow, J. D. Doyle, T. M. Uyeki, Influenza virus-related critical illness: Prevention, diagnosis, treatment. Crit. Care 23, 214 (2019). doi:10.1186/s13054019-2491-9 Medline

5. C. Wu, X. Chen, Y. Cai, J. Xia, X. Zhou, S. Xu, H. Huang, L. Zhang, X. Zhou, C. Du, Y. Zhang, J. Song, S. Wang, Y. Chao, Z. Yang, J. Xu, X. Zhou, D. Chen, W. Xiong, L. Xu, F. Zhou, J. Jiang, C. Bai, J. Zheng, Y. Song, Risk factors associated with acute respiratory distress syndrome and death in patients with coronavirus disease 2019 pneumonia in Wuhan, China. JAMA Intern. Med. (2020). 10.1001/jamainternmed.2020.0994 Medline

6. Q. Li, X. Guan, P. Wu, X. Wang, L. Zhou, Y. Tong, R. Ren, K. S. M. Leung, E. H. Y. Lau, J. Y. Wong, X. Xing, N. Xiang, Y. Wu, C. Li, Q. Chen, D. Li, T. Liu, J. Zhao, M. Liu, W. Tu, C. Chen, L. Jin, R. Yang, Q. Wang, S. Zhou, R. Wang, H. Liu, Y. Luo, Y. Liu, G. Shao, H. Li, Z. Tao, Y. Yang, Z. Deng, B. Liu, Z. Ma, Y. Zhang, G. Shi, T. T. Y. Lam, J. T. Wu, G. F. Gao, B. J. Cowling, B. Yang, G. M. Leung, Z. Feng, Early Transmission Dynamics in Wuhan, China, of Novel Coronavirus-Infected Pneumonia. N. Engl. J. Med. 382, 1199-1207 (2020). doi:10.1056/NEJMoa2001316 Medline

7. G. Grasselli, A. Zangrillo, A. Zanella, M. Antonelli, L. Cabrini, A. Castelli, D. Cereda, A. Coluccello, G. Foti, R. Fumagalli, G. Iotti, N. Latronico, L. Lorini, S. Merler, G. Natalini, A. Piatti, M. V. Ranieri, A. M. Scandroglio, E. Storti, M. Cecconi, A. Pesenti, A. Nailescu, A. Corona, A. Zangrillo, A. Protti, A. Albertin, A. Forastieri Molinari, A. Lombardo, A. Pezzi, A. Benini, A. M. Scandroglio, A. Malara, A. Castelli, A. Coluccello, A. Micucci, A. Pesenti, A. Sala, A. Alborghetti, B. Antonini, C. Capra, C. Troiano, C. Roscitano, D. Radrizzani, D. Chiumello, D. Coppini, D. Guzzon, E. Costantini, E. Malpetti, E. Zoia, E. Catena, E. Agosteo, E. Barbara, E. Beretta, E. Boselli, E. Storti, F. Harizay, F. Della Mura, F. L. Lorini, F. Donato Sigurtà, F. Marino, F. Mojoli, F. Rasulo, G. Grasselli, G. Casella, G. De Filippi, G. Castelli, G. Aldegheri, G. Gallioli, G. Lotti, G. Albano, G. Landoni, G. Marino, G. Vitale, G. Battista Perego, G. Evasi, G. Citerio, G. Foti, G. Natalini, G. Merli, I. Sforzini, L. Bianciardi, L. Carnevale, L. Grazioli, L. Cabrini, L. Guatteri, L. Salvi, M. Dei Poli, M. Galletti, M. Gemma, M. Ranucci, M. Riccio, M. Borelli, M. Zambon, M. Subert, M. Cecconi, M. G. Mazzoni, M. Raimondi, M. Panigada, M. Belliato, N. Bronzini, N. Latronico, N. Petrucci, N. Belgiorno, P. Tagliabue, P. Cortellazzi, P. Gnesin, P. Grosso, P. Gritti, P. Perazzo, P. Severgnini, P. Ruggeri, P. Sebastiano, R. D. Covello, R. Fernandez- 
Olmos, R. Fumagalli, R. Keim, R. Rona, R. Valsecchi, S. Cattaneo, S. Colombo, S. Cirri, S. Bonazzi, S. Greco, S. Muttini, T. Langer, V. Alaimo, U. Viola; CoVID-19 Lombardy ICU Network, Baseline Characteristics and Outcomes of 1591 Patients Infected With SARS-CoV-2 Admitted to ICUs of the Lombardy Region, Italy. JAMA 323, 1574 (2020). doi:10.1001/iama.2020.5394 Medline

8. P. Mehta, D. F. McAuley, M. Brown, E. Sanchez, R. S. Tattersall, J. J. Manson; HLH Across Speciality Collaboration, UK, COVID-19: Consider cytokine storm syndromes and immunosuppression. Lancet 395, 1033-1034 (2020). doi:10.1016/S0140-6736(20)30628-0 Medline

9. A. D. Amir, K. L. Davis, M. D. Tadmor, E. F. Simonds, J. H. Levine, S. C. Bendall, D. K. Shenfeld, S. Krishnaswamy, G. P. Nolan, D. Pe'er, viSNE enables visualization of high dimensional single-cell data and reveals phenotypic heterogeneity of leukemia. Nat. Biotechnol. 31, 545-552 (2013). doi:10.1038/nbt.2594 Medline

10. U. Müller, U. Steinhoff, L. F. Reis, S. Hemmi, J. Pavlovic, R. M. Zinkernagel, M. Aguet, Functional role of type I and type II interferons in antiviral defense. Science 264, 1918-1921 (1994). doi:10.1126/science.8009221 Medline

11. N. Jeremiah, B. Neven, M. Gentili, I. Callebaut, S. Maschalidi, M.-C. Stolzenberg, N. Goudin, M.-L. Frémond, P. Nitschke, T. J. Molina, S. Blanche, C. Picard, G. I. Rice, Y. J. Crow, N. Manel, A. Fischer, B. Bader-Meunier, F. Rieux-Laucat, Inherited STING-activating mutation underlies a familial inflammatory syndrome with lupus-like manifestations. J. Clin. Invest. 124, 5516-5520 (2014). doi:10.1172/JCl79100 Medline

12. M. P. Rodero, J. Decalf, V. Bondet, D. Hunt, G. I. Rice, S. Werneke, S. L. McGlasson, M.-A. Alyanakian, B. Bader-Meunier, C. Barnerias, N. Bellon, A. Belot, C. Bodemer, T. A. Briggs, I. Desguerre, M.-L. Frémond, M. Hully, A. M. J. M. van den Maagdenberg, I. Melki, I. Meyts, L. Musset, N. Pelzer, P. Quartier, G. M. Terwindt, J. Wardlaw, S. Wiseman, F. Rieux-Laucat, Y. Rose, B. Neven, C. Hertel, A. Hayday, M. L. Albert, F. Rozenberg, Y. J. Crow, D. Duffy, Detection of interferon alpha protein reveals differential levels and cellular sources in disease. J. Exp. Med. 214, 1547-1555 (2017). doi:10.1084/jem.20161451 Medline

13. P. Lebon, G. Ponsot, J. Aicardi, F. Goutières, M. Arthuis, Early intrathecal synthesis of interferon in herpes encephalitis. Biomedicine 31, 267-271 (1979). Medline

14. B. Reizis, Plasmacytoid Dendritic Cells: Development, Regulation, and Function. Immunity 50, 37-50 (2019). doi:10.1016/j.immuni.2018.12.027 Medline

15. Z. Zhou, L. Ren, L. Zhang, J. Zhong, Y. Xiao, Z. Jia, L. Guo, J. Yang, C. Wang, S. Jiang, D. Yang, G. Zhang, H. Li, F. Chen, Y. Xu, M. Chen, Z. Gao, J. Yang, J. Dong, B. Liu, X. Zhang, W. Wang, K. He, Q. Jin, M. Li, J. Wang, Overly Exuberant Innate Immune Response to SARS-CoV-2 Infection. (2020); available at http://dx.doi.org/10.2139/ssin.3551623.

16. S. F. Pedersen, Y.-C. Ho, SARS-CoV-2: A storm is raging. J. Clin. Invest. 130, 22022205 (2020). doi:10.1172/JCl137647 Medline

17. G. Chen, D. Wu, W. Guo, Y. Cao, D. Huang, H. Wang, T. Wang, X. Zhang, H. Chen, H. Yu, X. Zhang, M. Zhang, S. Wu, J. Song, T. Chen, M. Han, S. Li, X. Luo, J. Zhao, Q. Ning, Clinical and immunological features of severe and moderate coronavirus disease 2019. J. Clin. Invest. 130, 2620-2629 (2020). doi:10.1172/JC|137244 Medline

18. J. Rehwinkel, M. U. Gack, RIG-I-like receptors: Their regulation and roles in RNA sensing. Nat. Rev. Immunol. (2020). doi:10.1038/s41577-020-0288-3 Medline

19. S.-Y. Liu, D. J. Sanchez, R. Aliyari, S. Lu, G. Cheng, Systematic identification of type I and type II interferon-induced antiviral factors. Proc. Natl. Acad. Sci. U.S.A. 109, 4239-4244 (2012). doi:10.1073/pnas.1114981109 Medline

20. S. Heinonen, V. M. Velazquez, F. Ye, S. Mertz, S. Acero-Bedoya, B. Smith, E. Bunsow, C. Garcia-Mauriño, S. Oliva, D. M. Cohen, M. Moore-Clingenpeel, M. E. Peeples, O. Ramilo, A. Mejias, Immune profiles provide insights into respiratory syncytial virus disease severity in young children. Sci. Transl. Med. 12, eaaw0268 (2020). doi:10.1126/scitrans/med.aaw0268 Medline

21. D. Blanco-Melo, B. E. Nilsson-Payant, W.-C. Liu, S. Uhl, D. Hoagland, R. Møller, T. X. Jordan, K. Oishi, M. Panis, D. Sachs, T. T. Wang, R. E. Schwartz, J. K. Lim, R. A. Albrecht, B. R. tenOever, Imbalanced Host Response to SARS-CoV-2 Drives Development of COVID-19. Cell 181, 1036-1045.e9 (2020). doi:10.1016/i.cell.2020.04.026 Medline

22. E. Terán-Cabanillas, J. Hernández, Role of Leptin and SOCS3 in Inhibiting the Type I Interferon Response During Obesity. Inflammation 40, 58-67 (2017). doi:10.1007/s10753-016-0452-x Medline
23. E. Galkina, K. Ley, Immune and inflammatory mechanisms of atherosclerosis $\left({ }^{*}\right)$. Annu. Rev. Immunol. 27, 165-197 (2009). doi:10.1146/annurev.immunol.021908.132620 Medline

24. M. J. Ciancanelli, S. X. L. Huang, P. Luthra, H. Garner, Y. Itan, S. Volpi, F. G. Lafaille, C. Trouillet, M. Schmolke, R. A. Albrecht, E. Israelsson, H. K. Lim, M. Casadio, T. Hermesh, L. Lorenzo, L. W. Leung, V. Pedergnana, B. Boisson, S. Okada, C. Picard, B. Ringuier, F. Troussier, D. Chaussabel, L. Abel, I. Pellier, L. D. Notarangelo, A. García-Sastre, C. F. Basler, F. Geissmann, S.-Y. Zhang, H.-W. Snoeck, J.-L. Casanova, Infectious disease. Life-threatening influenza and impaired interferon amplification in human IRF7 deficiency. Science 348, 448-453 (2015). doi:10.1126/science.aaa1578 Medline

25. S. Clohisey, J. K. Baillie, Host susceptibility to severe influenza A virus infection. Crit. Care 23, 303 (2019). doi:10.1186/s13054-019-2566-7 Medline

26. L. Prokunina-Olsson, N. Alphonse, R. E. Dickenson, J. E. Durbin, J. S. Glenn, R. Hartmann, S. V. Kotenko, H. M. Lazear, T. R. O'Brien, C. Odendall, O. O. Onabajo, H. Piontkivska, D. M. Santer, N. C. Reich, A. Wack, I. Zanoni, COVID-19 and emerging viral infections: The case for interferon lambda. J. Exp. Med. 217, e20200653 (2020). doi:10.1084/jem.20200653 Medline

27. M. Roumier, R. Paule, M. Groh, A. Vallee, F. Ackermann, medRxiv, 2020.04.20.20061861 [Preprint] 22 April 2020. https://doi.org/10.1101/2020.04.20.20061861.

28. M. Feldmann, R. N. Maini, J. N. Woody, S. T. Holgate, G. Winter, M. Rowland, D. Richards, T. Hussell, Trials of anti-tumour necrosis factor therapy for COVID-19 are urgently needed. Lancet 395, 1407-1409 (2020). doi:10.1016/SO1406736(20)30858-8 Medline

29. P. Bost, A. Giladi, Y. Liu, Y. Bendjelal, G. Xu, E. David, R. Blecher-Gonen, M. Cohen, C. Medaglia, H. Li, A. Deczkowska, S. Zhang, B. Schwikowski, Z. Zhang, I. Amit, Host-Viral Infection Maps Reveal Signatures of Severe COVID-19 Patients. Cell 181, 1475-1488.e12 (2020). doi:10.1016/i.cell.2020.05.006 Medline

30. X. Yang, Y. Yu, J. Xu, H. Shu, J. Xia, H. Liu, Y. Wu, L. Zhang, Z. Yu, M. Fang, T. Yu, Y. Wang, S. Pan, X. Zou, S. Yuan, Y. Shang, Clinical course and outcomes of critically ill patients with SARS-CoV-2 pneumonia in Wuhan, China: A single-centered, retrospective, observational study. Lancet Respir. Med. 8, 475-481 (2020). doi:10.1016/S2213-2600(20)30079-5 Medline

31. J. Vandesompele, K. De Preter, F. Pattyn, B. Poppe, N. Van Roy, A. De Paepe, F. Speleman, Accurate normalization of real-time quantitative RT-PCR data by geometric averaging of multiple internal control genes. Genome Biol. 3, H0034 (2002). doi:10.1186/gb-2002-3-7-research0034 Medline

32. D. Rasch, F. Teuscher, V. Guiard, How robust are tests for two independent samples? J. Stat. Plan. Inference 137, 2706-2720 (2007). doi:10.1016/j.jspi.2006.04.011

33. M. E. R. Darnell, D. R. Taylor, Evaluation of inactivation methods for severe acute respiratory syndrome coronavirus in noncellular blood products. Transfusion 46 , 1770-1777 (2006). doi:10.1111/j.1537-2995.2006.00976.x Medline

\section{ACKNOWLEDGMENTS}

We acknowledge all health-care workers involved in the diagnosis and treatment of patients in Cochin Hospital, especially C. Azoulay L. Beaudeau, E. Canoui, P. Cohen, A. Contejean, B. Dunogué, D. Journois, P. Legendre, J. Marey and A. Régent. We thank Y. Gaudin for his advices on viral mechanism. We thank all the patients, supporters and our families for their confidence in our work. Funding: This study was supported by the Fonds IMMUNOV, for Innovation in Immunopathology. The study was also supported by the Institut National de la Santé et de la Recherche Médicale (INSERM) and the Institut Pasteur, by a government grant managed by the Agence National de la Recherche as part of the "Investment for the Future" program (ANR-10-IAHU-01 and the Laboratoire d'Excellence "Milieu Inteŕieur", grant no. ANR-10-LABX-69-01), and by a grant from the Agence National de la Recherche (ANR-flash Covid19 "AIROCovid" to FRL and "CoVarlmm" to DD), and by the FAST Foundation (French Friends of Sheba Tel Hashomer Hospital). J.H. is a recipient of an Institut Imagine MD-PhD fellowship program supported by the Fondation Bettencourt Schueller. L.B. is supported by the EUR G.E.N.E. (reference \#ANR-17-EURE-0013) program of the Université de Paris IdEX \#ANR-18-IDEX-0001 funded by the French Government through its "Investments for the Future" program. Author contributions: JH, NY, DD, FRL, SK and BT conceived and designed the study and had full access to 
all of the data in the study and take responsibility for the integrity of the data and the accuracy of the data analysis. JH, NY, AF, DD, FRL, SK and BT drafted the paper. JH, NY, LB, AC, JB, NS, DD, FRL, SK and BT did the analysis, and all authors critically revised the manuscript for important intellectual content and gave final approval for the version to be published. All authors agree to be

accountable for all aspects of the work in ensuring that questions related to the accuracy or integrity of any part of the work are appropriately investigated and resolved. Competing interests: We declare no competing interests. Data and materials availability: All data are available in the main text or the supplementary materials. This work is licensed under a Creative Commons Attribution 4.0 International (CC BY 4.0) license, which permits unrestricted use, distribution, and reproduction in any medium, provided the original work is properly cited. To view a copy of this license, visit

https://creativecommons.org/licenses/by/4.0. This license does not apply to figures/photos/artwork or other content included in the article that is credited to a third party; obtain authorization from the rights holder before using such material.

\section{SUPPLEMENTARY MATERIALS}

science.sciencemag.org/cgi/content/full/science.abc6027/DC1

Materials and Methods

Supplementary Text

Figs. S1 to S12

Tables S1 and S2

References (30-33)

3 May 2020; accepted 7 July 2020

Published online 13 July 2020

10.1126/science.abc6027 
A

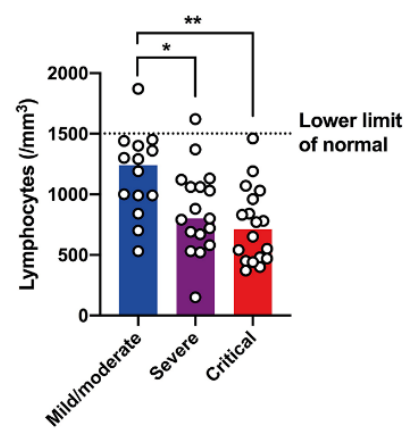

C

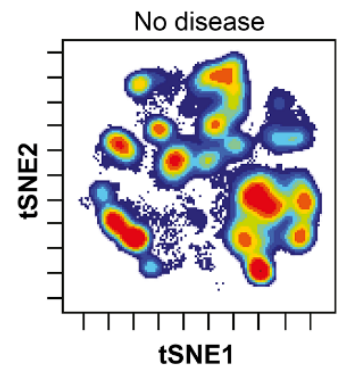

D

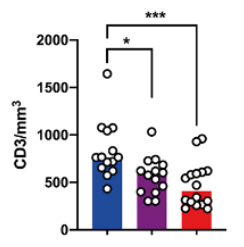

E
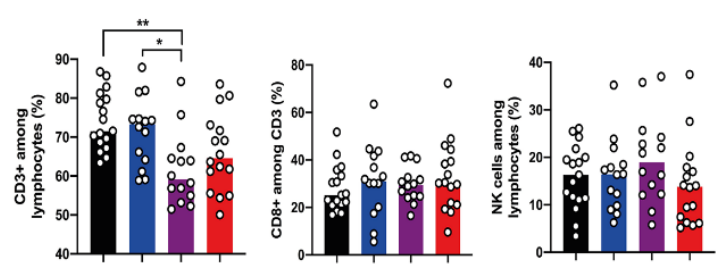

B
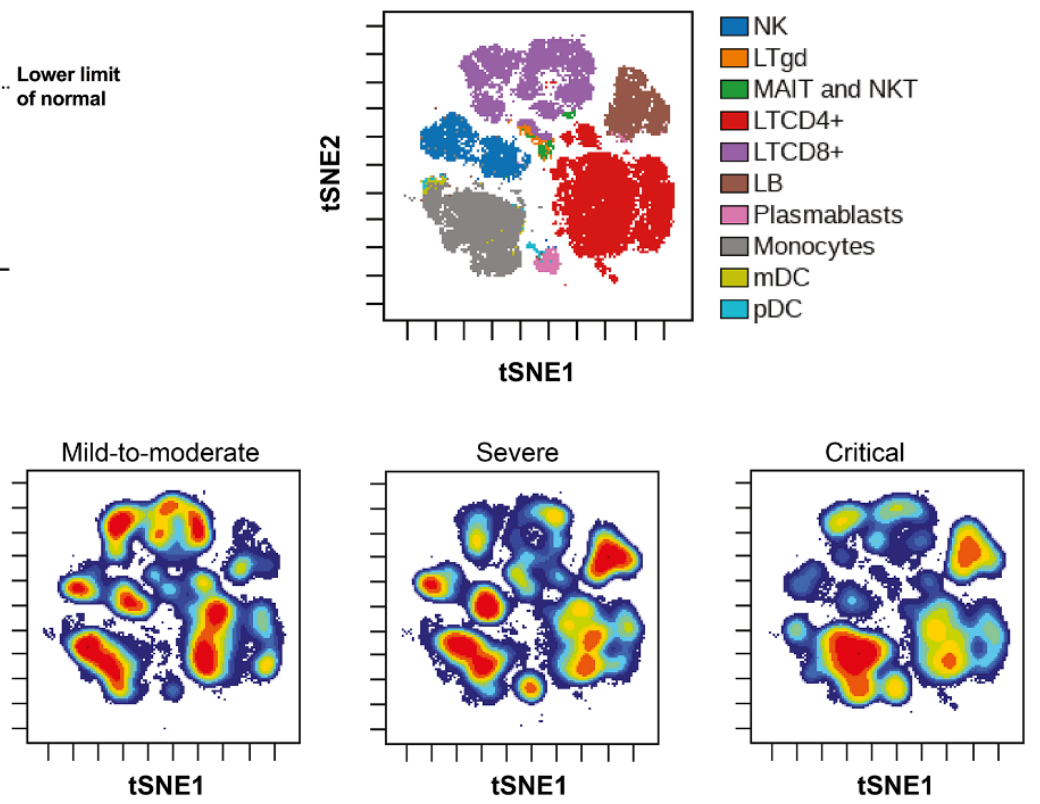

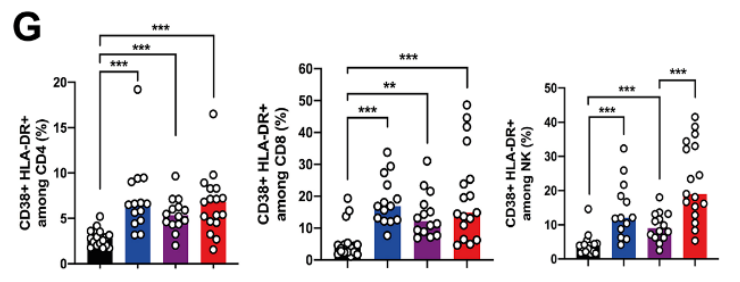

$\mathbf{F}$
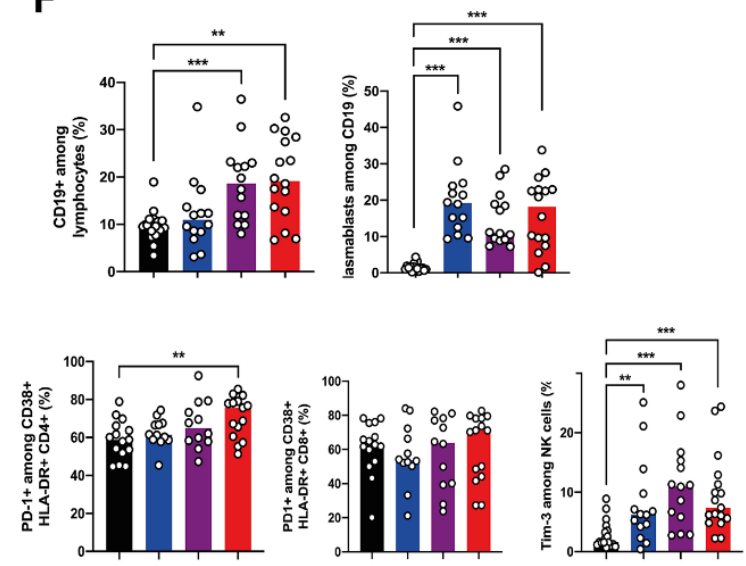
Fig. 1. Phenotyping of peripheral blood leukocytes in patients with SARS-CoV-2 infection. (A) Lymphocyte counts in whole blood from COVID-19 patients were analyzed between days 8 and 12 after onset of first symptoms, according to disease severity. (B) viSNE map of blood leukocytes after exclusion of granulocytes, stained with 30 markers and measured with mass cytometry. Cells are automatically separated into spatially distinct subsets based on the combination of markers that they express. (C) viSNE map colored by cell density across disease severity (classified as healthy controls, mild-to-moderate, severe and critical). Red represents the highest density of cells. (D) Absolute number of $\mathrm{CD}^{+} \mathrm{T}$ cells, $\mathrm{CD} 8^{+} \mathrm{T}$ cells and CD3- CD56 ${ }^{+}$natural killer (NK) cells in peripheral blood from COVID-19 patients, according to disease severity. (E and F) Proportions (frequencies) of lymphocyte subsets from COVID-19 patients. Shown are (E) proportions of CD3 ${ }^{+} \mathrm{T}_{\text {cells }}$ among lymphocytes, $\mathrm{CD}^{+} \mathrm{T}$ cells among $\mathrm{CD}^{+} \mathrm{T}$ cells and NK cells among lymphocytes; (F) proportions of $\mathrm{CD}_{19}{ }^{+} \mathrm{B}$ cells among lymphocytes and CD38hi CD27hi plasmablasts among CD19+ B cells. (G) Analysis of the functional status of specific T cell subsets and NK cells based on the expression of activation (CD38, HLA-DR) and exhaustion (PD-1, Tim-3) markers. In (D) to (G), data indicate median. Each dot represents a single patient. $P$ values were determined by the Kruskal-Wallis test, followed by Dunn's post-test for multiple group comparisons with median reported; ${ }^{*} P<0.05$; ${ }^{* *} P<0.01$; ${ }^{* *} P<0.001$. 

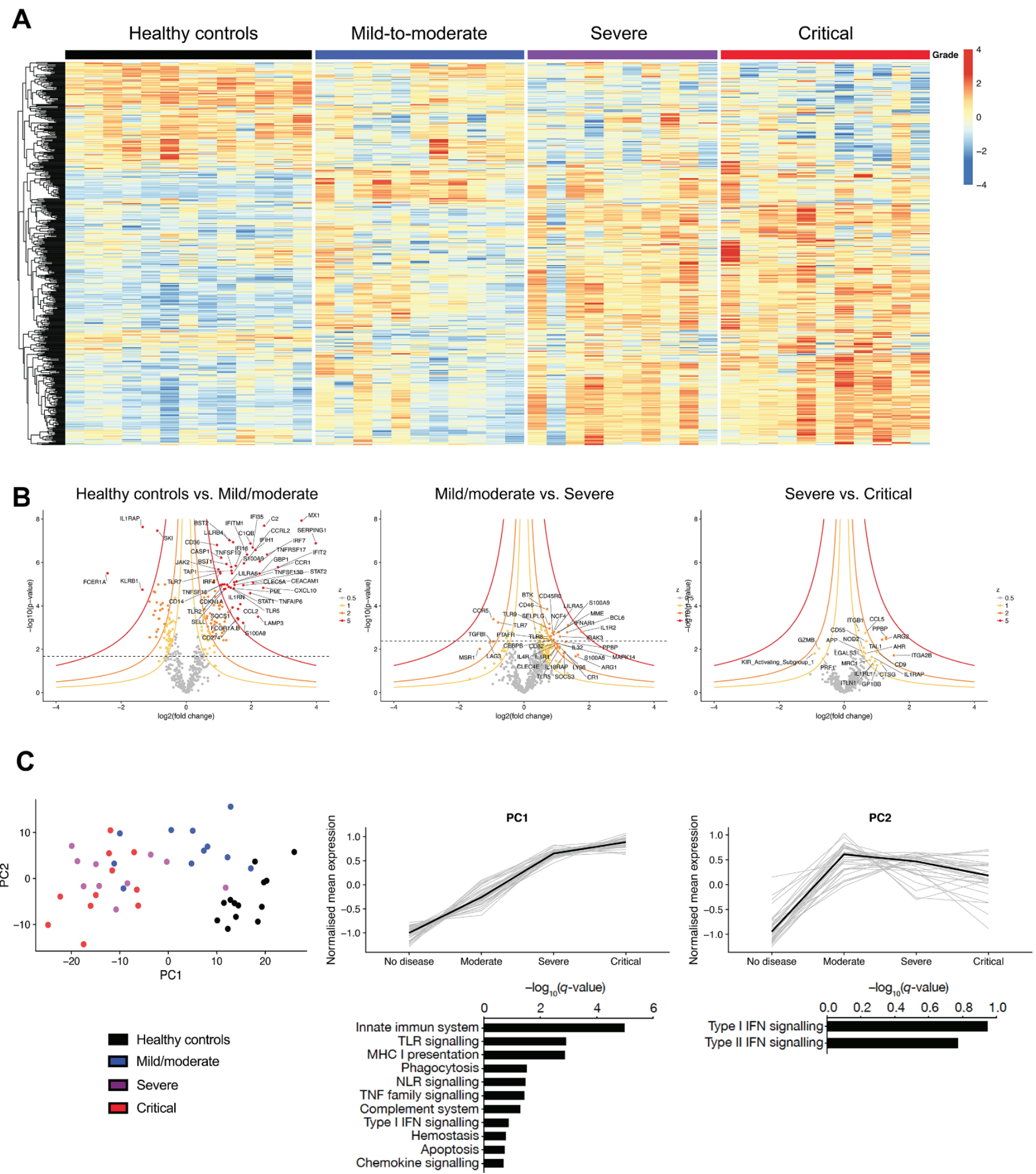
Type II IFN signalling

Mild/moderate

$\square$ Severe

$\square$ Critical

Chemokine signalling 
Fig. 2. Immunological transcriptional signature of SARS-CoV-2 infection. RNA extracted from patient whole blood and RNA counts of 574 genes were determined by direct probe hybridization using the Nanostring nCounter Human Immunology_v2 kit. (A) Heatmap representation of all genes, ordered by hierarchical clustering. Healthy controls $(n=13)$, mild-to-moderate $(n=11)$, severe $(n=10)$ and critical $(n=11)$. Upregulated genes are shown in red and down-regulated genes in blue. (B) Volcano plots depicting log10 ( $P$ value) and log2 (fold change), as well as z-value for each group comparison (see Methods). Gene expression comparisons allowed the identification of significantly differentially expressed genes between severity grades (heathy controls vs mild-moderate, 216 genes; moderate vs severe, 43 genes; severe vs critical, 0 genes). (C) Principal component analysis (PCA) of the transcriptional data (left). Kinetic plots showing mean normalized values for each gene and severity grade where each grey line corresponds to one gene (middle and right). Median values over genes for each severity grade are plotted in black. Gene set enrichment analysis of pathways enriched in PC1 and PC2 are depicted under corresponding kinetic plot. 
A
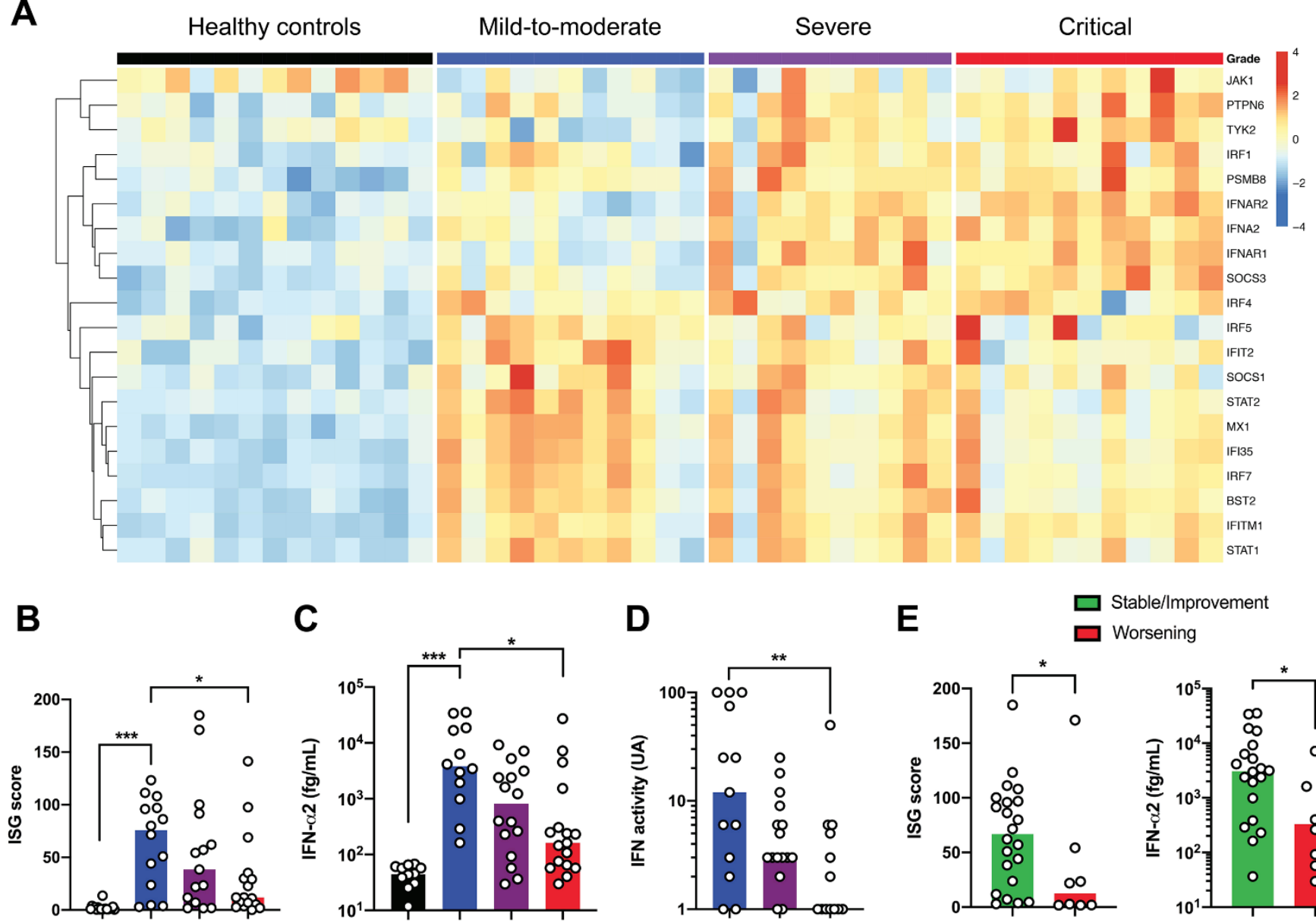

Stable/lmprovement
Worsening

$\mathbf{F}$

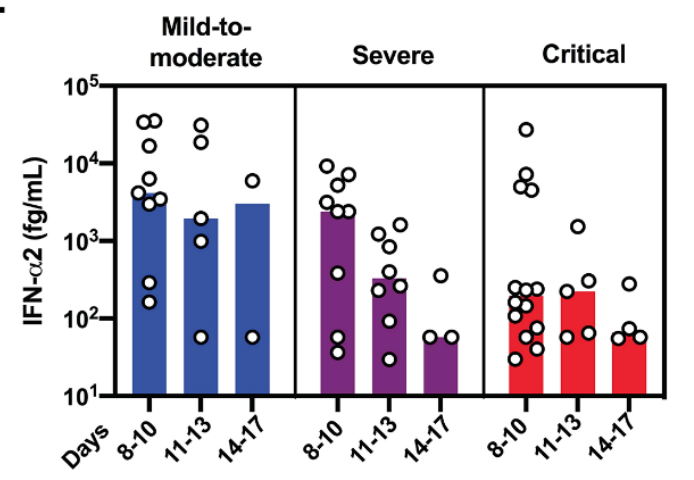

H

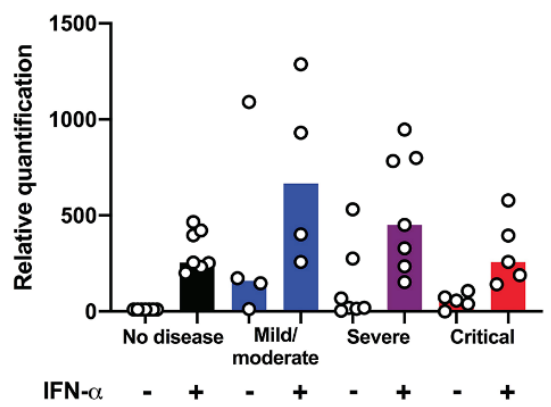

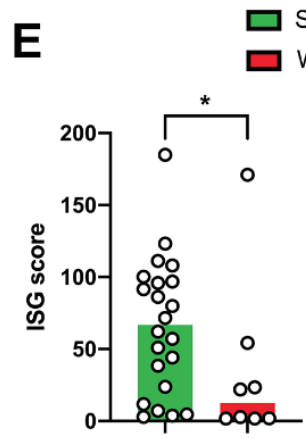

G
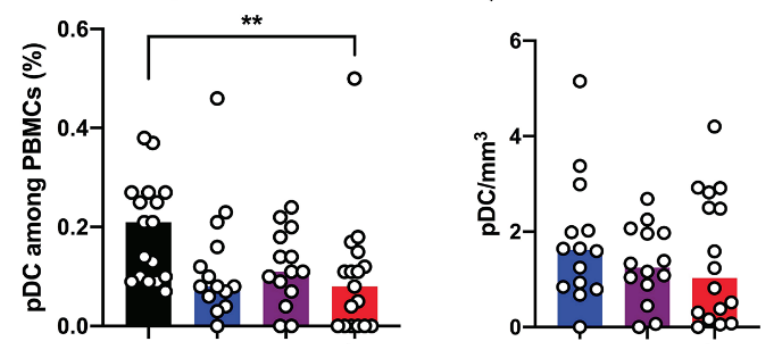

Detection of viral RNA

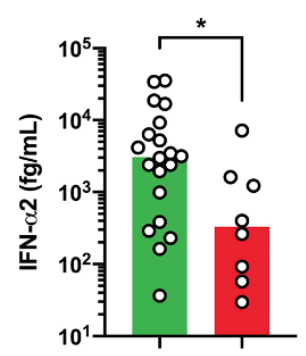

Healthy controls

\Mild/moderate

Severe

Critical
I

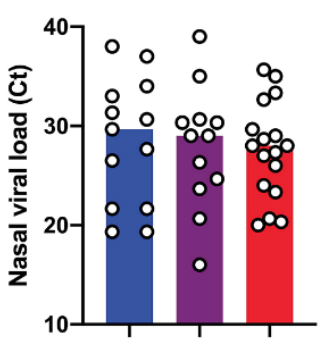

$\begin{array}{llll}0 \% & 60 \% & 76 \% & 88 \%\end{array}$

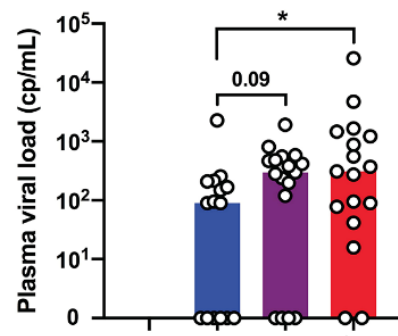


Fig. 3. Impaired type I IFN response in patients with severe SARS-CoV-2 infection. (A) Heatmap showing expression of type I IFN-related genes using the reverse transcription- and PCR-free Nanostring nCounter technology in patients with mild-to-moderate $(n=11)$, severe $(n=10)$ and critical $(n=11)$ SARS-CoV2 infection, and healthy controls $(n=13)$. Up-regulated genes are shown in red and down-regulated genes in blue. (B) IFN stimulated gene (ISG) score based on expression of 6 genes (IFI44L, IFI27, RSAD2, SIGLEC1, IFIT1, and IS15) measured by q-RT-PCR in whole blood cells from mild-to-moderate $(n=14)$, severe $(n=15)$ and critical $(n=17)$ patients, and healthy controls $(n=18)$. (C) IFN- $\alpha 2(\mathrm{fg} / \mathrm{mL})$ concentration evaluated by Simoa and (D) IFN activity in plasma according to clinical severity. (E) Mild-to-moderate $(n=14)$ and severe patients $(n=16)$ were separated in two groups depending on the clinical outcome, namely critical worsening requiring mechanical ventilation (to denote severe status). ISG score (left) and IFN- $\alpha 2$ plasma concentration (right) are shown. (F) Time-dependent IFN- $\alpha 2$ concentrations are shown according to severity group. (G) Quantification of plasmacytoid dendritic cells (pDC) as a percentage of PBMCs and as cells/mL according to severity group. (H) ISG score before and after stimulation of whole blood cells by IFN- $\alpha\left(10^{3} \mathrm{UI} / \mathrm{mL}\right.$ for 3 hours). (I) Viral loads in nasal swabs estimated by RT-PCR and expressed in cycle threshold (Ct) and blood viral load evaluated by digital PCR. In (B) and (E), ISG score results represent the fold-increased expression compared to the mean of unstimulated controls and are normalized to GAPDH. In (B) to (I), Data indicate median. Each dot represents a single patient. $P$-values were determined by the Kruskal-Wallis test, followed by Dunn's post-test for multiple group comparisons and by the Mann-Whitney test for two group comparisons with median reported; * $P<0.05$; ** $P<0.01 ;{ }^{* * *} P<0.001$. 


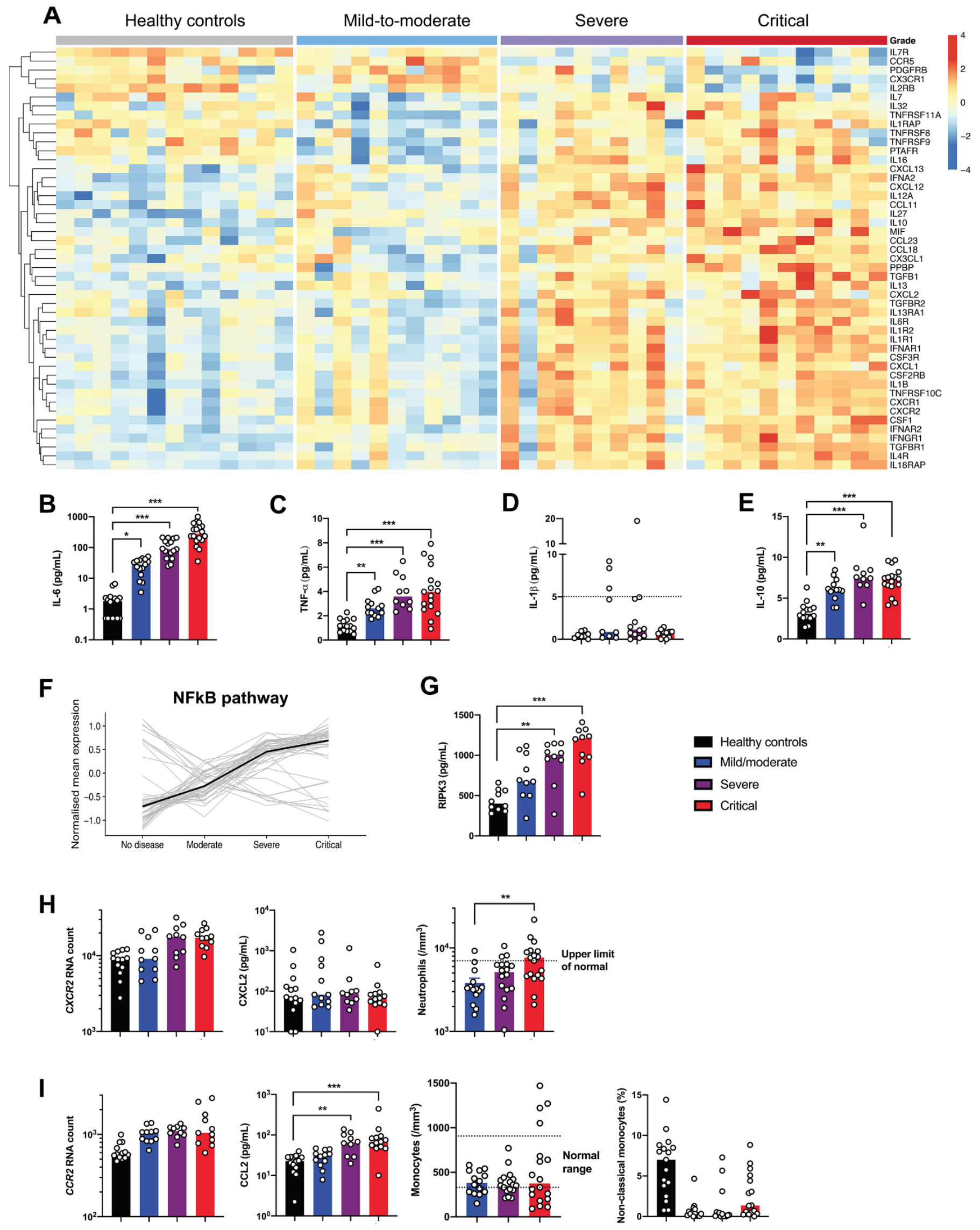


Fig. 4. Immune profiling in patients with severe and critical SARS-CoV-2 infection. (A) Heatmap showing the expression of cytokines and chemokines that are significantly different in severe and critical patients, and ordered by hierarchical clustering. Healthy controls $(n=13)$, mild-to-moderate $(n=11)$, severe $(n=10)$ and critical $(n=11)$ patients. Up-regulated genes are shown in red and down-regulated genes in blue. (B) Interleukin (IL)-6, (C) Tumor necrosis factor (TNF)- $\alpha$, (D) IL-1 $\beta$ and (E) IL-10 proteins were quantified in the plasma of patients using Simoa technology or a clinical grade ELISA assay (see methods). Each group includes $n=10-18$ patients. Dashed line depicts the limit of detection (LOD). (F) Kinetic plots showing mean normalized value for each gene and severity grade (each grey line corresponds to one gene belonging to the NF-kB pathway). Median values over genes for each severity grade were plotted in black. (G) Plasma quantification of receptor-interacting protein kinase (RIPK)3. Each group included $n=10$ patients. $(\mathrm{H})$ Absolute RNA count for CXCR2 (left); CXCL2 protein plasma concentration measured by Luminex technology (middle); blood neutrophil count depending on severity group (right). Dashed line depicts the upper normal limit. Each group includes $n=10-13$ patients (I) Absolute RNA count for CCR2 (left); CCL2 protein plasma concentration measured by Luminex technology (middle left); blood monocyte count depending on severity group (middle right). Dashed lines depict the normal range. (Right) The percentage of non-classical monocytes depending on severity grade. Each group shows $n=10-18$ patients. RNA data are extracted from the Nanostring nCounter analysis (see methods). In (B) to (I), data indicate median. Each dot represents a single patient. $P$-values were determined by the Kruskal-Wallis test, followed by Dunn's post-test for multiple group comparisons with median reported; ${ }^{*} P<0.05$; ${ }^{* *} P<0.01$; ${ }^{* *} P<0.001$. 